\title{
The pulsating $\lambda$ Bootis star 15 Andromedae: results from a three-site photometry campaign $\star$ (Research Note)
}

\author{
T. N. Dorokhova ${ }^{1}$, G. Handler ${ }^{2}$, N. I. Dorokhov ${ }^{1}$, E. Rodriguez ${ }^{3}$, and S. N. Udovichenko ${ }^{1}$ \\ 1 Astronomical Observatory of Odessa National University, Marazlievskaya 1v, 65014 Odessa, Ukraine \\ e-mail: tnd@te.net.ua \\ 2 Institut für Astronomie, Universität Wien, Türkenschanzstrasse 17, 1180 Wien, Austria \\ e-mail: handler@astro.univie.ac.at \\ 3 Instituto de Astrofisica de Andalucia, C.S.I.C., Apdo. 3004, 18080 Granada, Spain
}

Received 27 September 2007 / Accepted 29 November 2007

\section{ABSTRACT}

\begin{abstract}
Context. The $\lambda$ Bootis stars are a chemically peculiar subgroup of main sequence A/F stars. Several of these fall into the instability strip of the $\delta$ Scuti stars and the whole spectroscopic group shows a high incidence of pulsational variability.

Aims. We investigated the pulsational behaviour of the $\lambda$ Bootis star 15 And (HD 221756) to determine as many intrinsic mode frequencies as possible for asteroseismic applications.

Methods. The star was included as a secondary target for three telescopes during a campaign devoted to the $\beta$ Cep stars 12 and 16 Lac, due to its proximity to these targets. $302.8 \mathrm{~h}$ of time-resolved photometry spanning $161.2 \mathrm{~d}$ were acquired and analyzed.

Results. Our frequency analysis revealed $f_{1}=24.7887(2) \mathrm{c} / \mathrm{d}$ as the main pulsation frequency, with an amplitude of only $1.9(1) \mathrm{mmag}$. The residual periodogram suggests the presence of additional frequencies in an interval from 20 to $25 \mathrm{c} / \mathrm{d}$, with very low amplitudes. We succeeded in detecting a second frequency $f_{2}=22.2580(9)$ with an amplitude of 0.6(1) mmag. Low-frequency light variations of the comparison star HD 220575 were revealed as well.

Conclusions. The pulsational behaviour of 15 And as a typical $\lambda$ Boo-type star is compared with that of $\delta$ Scuti stars with normal abundances and with metallic-line $\delta$ Scuti stars. We discuss the value of $\lambda$ Boo stars in understanding accretion and mixing in pulsating stars. The difficulties in the selection of reliable comparison stars for such high-precision research are pointed out.
\end{abstract}

Key words. stars: chemically peculiar - stars: individual: HD 221756 - stars: individual: HD 220575 - stars: oscillations stars: variables: $\delta$ Scuti

\section{Introduction}

\section{1. $\lambda$ Bootis stars and their pulsations}

Paunzen (2004) defined the $\lambda$ Boo-type stars as "Population I late B to early F stars, with moderate to extreme (up to a factor 100) surface underabundances of most Fe-peak elements and solar abundances of lighter elements (C, N, O and S)".

The group is very not numerous: the list of the stars consisted of seven members until the late 1980s. Later on, Gray (1988) described 20 stars. Presently, about $60 \lambda$ Bootis stars are known and have been most recently listed by Paunzen et al. (2002a). This means that no more than $2 \%$ of galactic field stars in the spectral range B8-F4 belong to the $\lambda$ Bootis stars (Paunzen et al. 2002a; Gray \& Corbally 1993). If the $\lambda$ Bootis phenomenon corresponded to a specific phase of stellar evolution, this would mean that this stage exists for no longer than $10^{6}$ years.

This is exactly the time scale on which diffusion is expected to destroy any peculiar abundance pattern acquired by a nonmagnetic A-type star (Turcotte \& Charbonneau 1993). Kamp \& Paunzen (2002) proposed a scenario that explains all the basic

\footnotetext{
* Individual photometry measurements are only available in electronic form at the CDS via anonymous ftp to cdsarc.u-strasbg. fr $(130.79 .128 .5)$ or via

http://cdsweb.u-strasbg.fr/cgi-bin/qcat?J/A+A/480/187
}

characteristics of the $\lambda$ Bootis stars: the peculiar abundance pattern is caused by the interaction of a star and a diffuse interstellar cloud.

In the HR diagram $\lambda$ Bootis stars fall inside the classical instability strip. Paunzen et al. (2002b, hereinafter PHW) estimated that at least $70 \%$ of the group members pulsate, often with high overtone modes. Thus intensive spectroscopic and photometric investigations of their $\delta$ Sct-type pulsational activity are of special interest for asteroseismology.

However, the amplitudes of these oscillations are usually low, probably due to the large rotational velocities of most $\lambda$ Bootis stars. This is perhaps the reason why only some stars have been investigated with multi-site observations (Paunzen et al. 1998; Martinez et al. 1998; Breger et al. 2006; Mkrtichian et al. 2007).

\subsection{And $=H D 221756$}

15 And (HD 221756, HR 8947, V340 And) was suspected to be variable by Rufener \& Bartholdi (1982) who found an rms scatter of 24 mmag in their Geneva V measurements of this star.

Abt (1984) included 15 And in his list of $\lambda$ Boo candidates. Gray (1988) confirmed the $\lambda$ Boo features in its spectrum and classified it as a transitional object between peculiar and normal types of hydrogen line cores. Stürenburg (1993) derived 
Table 1. The information for the target and comparison stars of the campaign.

\begin{tabular}{lcccl}
\hline \hline \multicolumn{1}{c}{ Star } & $\alpha_{2000}$ & $\delta_{2000}$ & $m_{v}$ & \multicolumn{1}{c}{ Sp } \\
\hline V1 12 Lac & 224129 & +40133 & 5.23 & B2III \\
C1 $=10 \mathrm{Lac}$ & 223916 & +390301 & 4.88 & O9V \\
V2 $=16 \mathrm{Lac}$ & 225624 & +413614 & 5.58 & B2IV \\
C2 $=2$ And & 230236 & +424528 & 5.09 & A3Vn \\
V3 $=15$ And & 233438 & +401411 & 5.56 & A1III \\
C3= HD 220575 & 232435 & +410646 & 6.72 & B8III \\
\hline
\end{tabular}

abundances of $\mathrm{Fe}, \mathrm{Mg}$, $\mathrm{Ti}, \mathrm{Cr}$ and $\mathrm{Ba}$ and revealed metal deficiencies of the order of 0.5 dex below the solar values. Gray \& Corbally (1993) classified the spectrum of HD 221756 as A1 $\mathrm{Va}+$ and included the star in their list of "bona fide" $\lambda$ Boo stars.

Paunzen \& Handler (1996) tested HD 221756 for pulsations and detected a period of $63 \mathrm{~min}$ with a peak-to-peak amplitude of 6.6 mmag in Strömgren b. Dorokhova \& Dorokhov (1997) confirmed this conclusion and suspected multiperiodic variability of the star.

Bohlender et al. (1999) have specified 15 And as a mild $\lambda$ Boo star and revealed circumstellar features in the Na I D lines during a detailed inspection of its spectrum. These authors also determined the projected rotation velocity of the star as $105 \mathrm{~km} \mathrm{~s}^{-1}$, which is in good agreement with previous results.

Bohlender et al. (1999) also detected nonradial pulsations (NRP) with a characteristic time scale of about $31 \mathrm{~min}$ and an amplitude of approximately $1 \%$ of the continuum level using the TiII, FeII and NaI D lines. However, the authors noticed a discrepancy between their estimates of the NRP periods and those from photometry. Thus, a more precise specification of the star's oscillation behaviour is desirable.

During the preparation of a multisite campaign for the $\beta$ Cephei stars 12 and 16 Lac (Handler et al. 2006), we noticed that 15 And is located near these objects. We added the star as a secondary target only for some telescopes because the main program was rather complicated already: four stars observed with 3 filters. In Table 1 the coordinates, magnitudes, spectral and luminosity classes are given for all target and comparison stars of the campaign.

\section{Observations}

The multi-site campaign took place from August 2003 to January 2004, using photoelectric photometers and the "threestar method" (Breger 1992) that was in this case extended to a "six-star method". From experience with previous observations, HD 220575 was selected as a local comparison star for 15 And. HD 220575 is a HgMn star (see, for example, Wolf \& Wolf 1974). This peculiarity to a certain degree is "an assurance of quality" for the comparison star, because photometric variability of such stars is rare. We could also use the other two comparison stars of the campaign for reference.

The $0.8 \mathrm{~m}$ Ritchey-Chretien telescope (DE) of the Astronomical Observatory of Odessa National University at Mt. Dushak-Erekdag Observatory in Turkmenistan (Dorokhov \& Dorokhova 1994) was used first. The photometry was obtained with the Johnson $V$ filter, the Nyquist frequency was $66.73 \mathrm{c} / \mathrm{d}$, and observations were acquired for $82.08 \mathrm{~h}$.

The main part of the observations was done with the $0.75 \mathrm{~m}$ Austrian Automatic Photoelectric Telescope (APT) at Fairborn Observatory, Arizona (Strassmeier et al. 1997). The APT carried out automatic observations of six stars, four with the Strömgren uvy filters and HD 221756 and the comparison star HD 220575 with the $y$ filter, resulting in a Nyquist frequency of $58.38 \mathrm{c} / \mathrm{d}$ and a total time of observation of $209.06 \mathrm{~h}$.

The simultaneous six-channel $u v b y \beta$ photometer (Nielsen 1983 ) attached to the Sierra-Nevada $0.9 \mathrm{~m}$ telescope (OSN) was used during 3 nights (11.65 h) of September for multicolour photometry with a Nyquist frequency of $417.8 \mathrm{c} / \mathrm{d}$.

In total we obtained a data set of $303.79 \mathrm{~h}$ of measurement spanning a time base of $161.2 \mathrm{~d}$. The observing log is given in Table 2. No overlaps between different sites occurred during the campaign. The data were corrected for coincidence counting losses, sky background contribution and atmospheric extinction. The time of each observation was transformed to Heliocentric Julian Date. Figure 1 shows the longest differential light curves obtained at each observatory. The diameters of the plot symbols approximately correspond to the standard errors of the data. The OSN data were binned to a similar Nyquist frequency as the data from the other sites.

\section{The frequency analysis}

For the frequency analysis of the differential light curve we used the program packages PERIOD (Breger 1990), PERIOD98 (Sperl 1998) and PERIOD04 (Lenz \& Breger 2005). As a first step we obtained Fourier spectra of each site's data. All three periodograms showed a dominant frequency at about $24.8 \mathrm{c} / \mathrm{d}$ and low-frequency variability.

Since variability of comparison stars was revealed in many campaigns (see, for example, Handler et al. 2003, 2004), we at first reduced the light curves of 15 And and HD 220575 relative to the non-local comparison stars, $10 \mathrm{Lac}$ and 2 And (see Table 1). This analysis showed that only 10 Lac did not introduce its own variability to the differential data.

\subsection{The problem of comparison stars}

The Fourier spectrum of the most extensive data set (from the APT) of 15 And relative to HD 220575 has the strongest peak at $f=24.788 \mathrm{c} / \mathrm{d}$ with an amplitude of about $1.6 \mathrm{mmag}$, and some peaks at low frequencies with a maximum amplitude of 1.3 mmag (Fig. 2a). In comparison, the Fourier spectrum of 15 And relative to 10 Lac (Fig. 2b) shows the same $f=24.788 \mathrm{c} / \mathrm{d}$ peak with a somewhat larger amplitude (about $1.8 \mathrm{mmag}$ ) due to the higher noise level. The higher noise, especially at low frequencies, compared to the 15 And - HD 220575 data arises from the colour and air mass differences of those two stars compared to $10 \mathrm{Lac}$.

The amplitude spectrum of $\mathrm{C} 3=\mathrm{HD} 220575$ relative to $\mathrm{C} 1=10 \mathrm{Lac}$ (Fig. 2c) shows the same pattern at low frequencies as Fig. 2a. The strongest peak has $f=1.1 \mathrm{c} / \mathrm{d}$ and an amplitude of about $1.2 \mathrm{mmag}$. We therefore suspect that HD 220575 is itself variable, but cannot fully prove this from our data.

For the final analysis we used the differential photometry 15 And relative to HD 220575 as the closest comparison star. We assumed that the latter is variable, and removed its variability prior to frequency analysis.

\subsection{Analysis of the total data set}

The data from all observatories were merged. The Strömgren $y$ and Johnson $V$ filter data were treated as equivalent because their effective wavelength is very similar (see Handler 1998). The Fourier spectrum of the merged data is presented in Fig. 3. 
Table 2. Journal of observations of 15 And.

\begin{tabular}{|c|c|c|c|c|c|c|c|c|c|}
\hline$\overline{\text { HJD }_{b}}$ & Length $(h)$ & 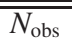 & Site & $\overline{\mathrm{HJD}_{b}}$ & Length $(h)$ & $\overline{N_{\text {obs }}}$ & $\overline{\text { HJD }_{b}}$ & Length $(h)$ & 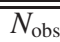 \\
\hline 53.2585 & 5.88 & 22 & $\mathrm{DE}$ & 118.5968 & 7.10 & 35 & 153.5685 & 2.74 & 11 \\
\hline 54.2415 & 5.89 & 20 & & 123.6925 & 4.52 & 18 & 158.5665 & 2.46 & 13 \\
\hline 56.2301 & 6.50 & 27 & & 124.5917 & 6.20 & 21 & 163.5650 & 2.05 & 11 \\
\hline 60.2143 & 6.78 & 27 & & 125.5909 & 6.84 & 28 & 164.5647 & 2.05 & 11 \\
\hline 61.2344 & 6.70 & 30 & & 126.6453 & 5.50 & 27 & 165.5644 & 1.97 & 10 \\
\hline 62.2560 & 6.03 & 23 & & 127.6860 & 4.43 & 22 & 166.5641 & 1.82 & 9 \\
\hline 63.2242 & 6.65 & 18 & & 129.5836 & 6.69 & 33 & 167.5639 & 1.85 & 10 \\
\hline 64.2050 & 6.79 & 29 & & 130.5828 & 6.60 & 32 & 170.5632 & 1.58 & 8 \\
\hline 65.2181 & 6.62 & 23 & & 131.5821 & 6.67 & 32 & 171.5632 & 1.61 & 7 \\
\hline 66.2241 & 6.95 & 31 & & 132.5813 & 6.49 & 32 & 190.5637 & 3.12 & 14 \\
\hline 67.2085 & 2.60 & 16 & & 133.5806 & 6.49 & 32 & 191.5641 & 2.99 & 15 \\
\hline 70.3640 & 2.79 & 15 & & 134.5796 & 6.48 & 32 & 192.6508 & 0.94 & 5 \\
\hline 71.1992 & 7.63 & 36 & & 135.5792 & 6.49 & 32 & 194.5647 & 2.79 & 14 \\
\hline 75.3421 & 4.27 & 21 & & 136.5782 & 6.28 & 31 & 195.5684 & 2.60 & 12 \\
\hline 95.3511 & 6.05 & 10 & OSN & 137.5862 & 6.07 & 23 & 198.5658 & 2.58 & 13 \\
\hline 96.3374 & 2.65 & 81 & & 138.5772 & 6.18 & 29 & 199.5663 & 2.38 & 12 \\
\hline 99.5581 & 2.94 & 69 & & 139.5765 & 6.28 & 31 & 200.6453 & 0.52 & 3 \\
\hline 111.8311 & 1.96 & 10 & APT & 141.6607 & 4.02 & 20 & 201.5669 & 0.82 & 5 \\
\hline 112.6020 & 7.31 & 36 & & 145.6070 & 2.26 & 12 & 202.6273 & 0.73 & 4 \\
\hline 113.6010 & 7.24 & 33 & & 147.6739 & 0.46 & 2 & 204.5681 & 0.82 & 5 \\
\hline 114.6002 & 7.27 & 35 & & 148.5905 & 2.46 & 13 & 206.6008 & 0.61 & 4 \\
\hline 115.6078 & 7.10 & 26 & & 149.5825 & 2.67 & 14 & 207.5693 & 1.85 & 9 \\
\hline 116.5983 & 7.10 & 34 & & 150.5794 & 2.63 & 13 & 211.5832 & 1.14 & 6 \\
\hline 117.5976 & 7.10 & 35 & & 152.6463 & 0.82 & 5 & 214.5721 & 1.34 & 7 \\
\hline
\end{tabular}

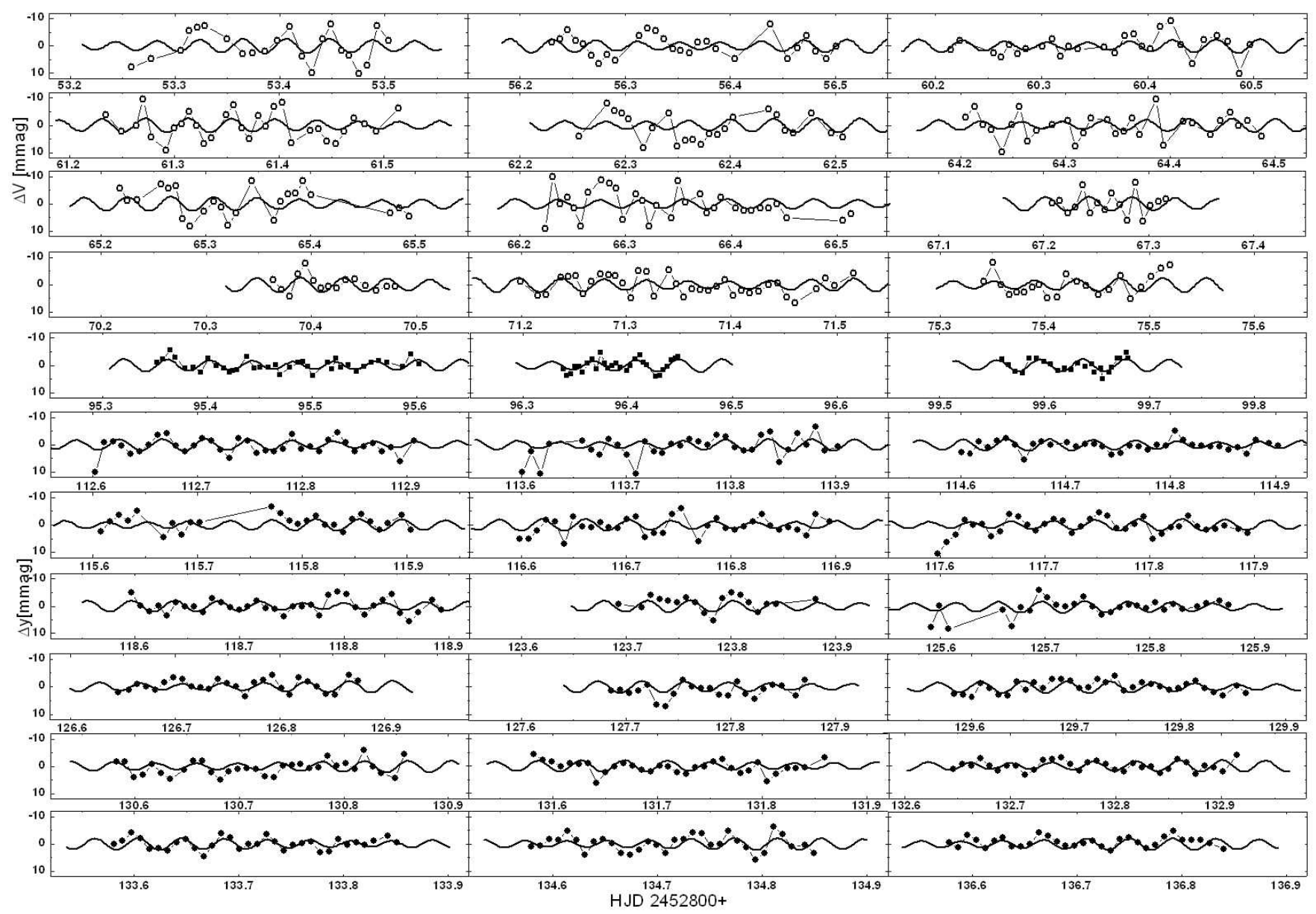

Fig. 1. The differential photometry obtained at each observatory (DE - open circles, OSN - squares, APT - filled circles) normalized to zero. The solid line is a least squares fit of two frequencies, which were revealed from subsequently prewhitened amplitude Fourier spectra of the combined data. 


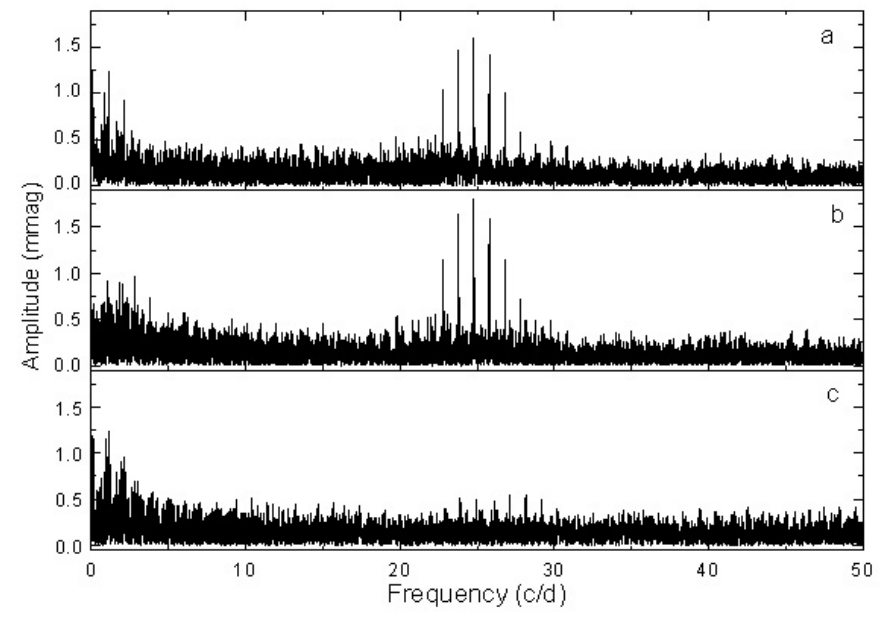

Fig. 2. Strömgren $y$-filter Fourier spectra: a) 15 And relative to HD 220575 ; b) 15 And relative to $10 \mathrm{Lac}$; c) HD 220575 relative to 10 Lac.

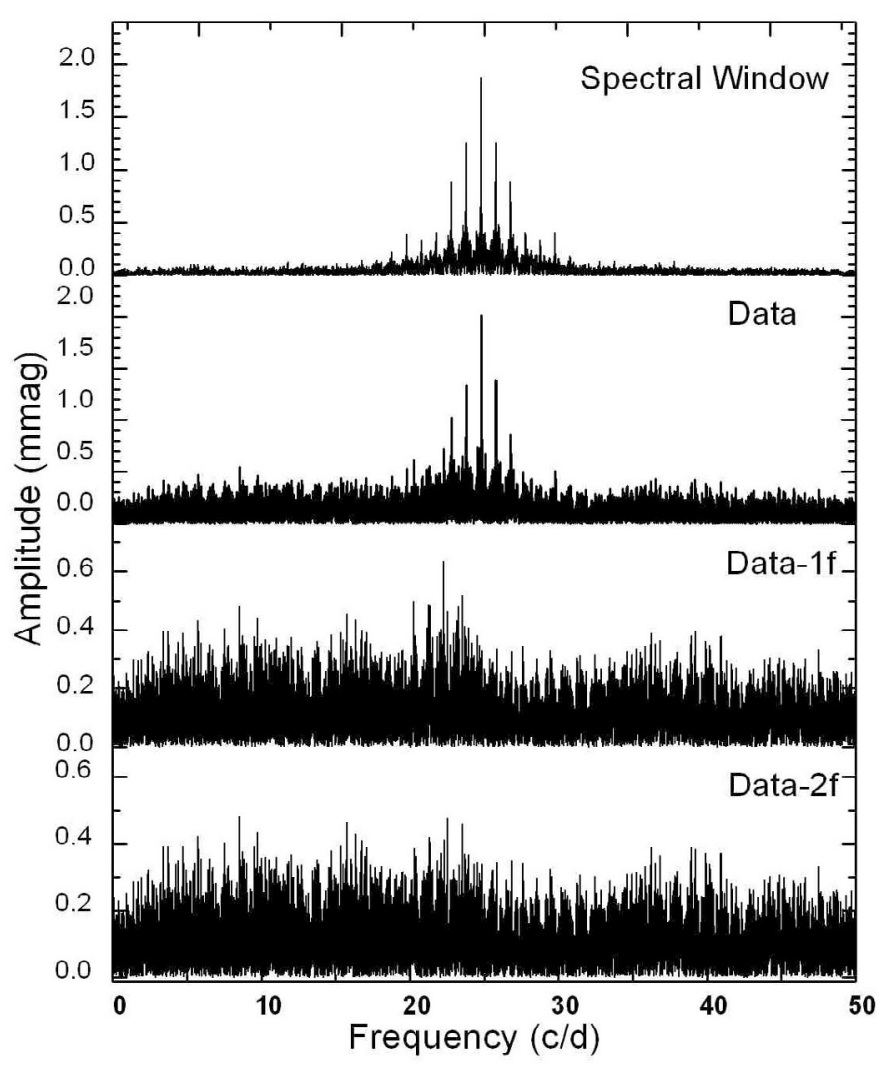

Fig. 3. Fourier amplitude spectrum of the combined data in the $V$ and $y$ filters. Some prewhitening steps are indicated.

The principal frequency $f_{1}$ is clearly present and was prewhitened. The residual amplitude spectrum (third panel of Fig. 3) shows another frequency, $f_{2}$. We consider $f_{2}$ significant because it exceeds the noise level by a factor larger than four, following Breger et al. (1993).

After prewhitening of two frequencies from the 1588 measurements, the residual spectrum reveals the possible presence of some additional signals in the narrow region of 22 to $25 \mathrm{c} / \mathrm{d}$ but their amplitudes are too low for further analysis. Our twofrequency solution is shown in Table 3.
Table 3. The multifrequency solution for the total campaign data set.

\begin{tabular}{cccc}
\hline \hline ID & Frequency (c/d) & Amplitude (mmag) & $S / N$ \\
\hline$f_{1}$ & $24.7887 \pm 0.0002$ & $1.9 \pm 0.1$ & 14.5 \\
$f_{2}$ & $22.2580 \pm 0.0009$ & $0.6 \pm 0.1$ & 4.6 \\
\hline
\end{tabular}

Our measurements are consistent with the initial results of Paunzen \& Handler (1996) within $\sim 2 \sigma$ : adding formal error estimates as derived by Montgomery \& O'Donoghue (1999), we find a frequency of $22.9 \pm 0.9 \mathrm{c} / \mathrm{d}$ and a Strömgren $b$ semiamplitude of $3.3 \pm 0.7 \mathrm{mmag}$ in these discovery data.

\section{Summary and discussion}

The slow variability initially suspected for 15 And is most likely due to the comparison star HD 220575 probably being variable at the mmag level with a period near $1 \mathrm{~d}$.

As already mentioned, HD 220575 as a HgMn star is unlikely to be variable. However, the star is also a spectroscopic binary (Schneider 1981) and its variability might be associated with binarity. However, from the very low amplitude and diurnal breaks in our mostly single-colour data we cannot pinpoint the type of variability HD 220575 might exhibit.

15 And is one of the hottest known $\delta$ Scuti pulsators (see PHW) and may even be located outside the hot border of the classical instability strip in the HR Diagram. However, this hot border is observationally still not very well defined. Some chemically normal stars that may cause a revision of this instability strip border to higher temperatures are discussed by Rodriguez $\&$ Breger (2001). The question of whether $\lambda$ Boo stars can show observable pulsations at higher temperatures than stars with normal chemical abundances is therefore still open, and more observational work needs to be done to define the hot border of the $\delta$ Scuti strip precisely. This is the first multi-site campaign for such a low-amplitude star.

We estimated the pulsational constants of the oscillations of 15 And using $\log T_{\text {eff }}=3.93$ and $\log g=3.9$ from PHW. $Q_{1}=0.013 \mathrm{~d}$ was derived for the first period $P_{1}=0.0403 \mathrm{~d}$ and $Q_{2}=0.015 \mathrm{~d}$ conforms to the second period $P_{2}=0.0449 \mathrm{~d}$. Both $Q$ values are within the most common range for pulsating $\lambda$ Boo stars (Fig. 6 from PHW) corroborating that 15 And is a representative member of the class.

Low-amplitude multiperiodicity is a typical feature of $\lambda$ Boo stars as well as of rapidly rotating $\delta$ Sct stars. The pulsational properties of this group are not different from "normal" $\delta$ Scuti stars and even from some Am pulsators, e.g. HR 2100 (V1004 Ori) with parameters $\log T_{\text {eff }}=3.883, \log g=3.64$, $Q=0.0124$ d determined by Liu (1999).

This is in agreement with the recent conclusion made by Breger et al. (2006) from a comparison of the pulsation spectrum of HD 210111, the richest of a $\lambda$ Bootis star known to date, with that of some other $\delta$ Sct stars located in a similar range of the HR diagram. This indicates that the driving mechanism of the pulsations is the same for all groups, which is substantiated by theoretical calculations (Turcotte 2002, 2005).

Generally the $\lambda$ Boo stars are very complicated objects for spectral (extremely broad and weak lines) and photometric (extremely low amplitudes and unstable modes) investigations. Precise space observations may provide sufficient information to perform theoretical calculations at a level of precision that makes seismic sounding of these objects possible.

Acknowledgements. This research has used databases of the Simbad, the Canadian Astronomy Data Centre (CADC), the Vienna Selection of 
Astronomical Targets (VISAT). TND thanks Prof. Sergey Andriyevsky and Dr. Sylvain Turcotte for valuable discussions. GH is supported by the Austrian Fonds zur Förderung der wissenschaftlichen Forschung under grant P18339-N08. We are grateful to the referee, Dr. David Bohlender, for his constructive remarks that helped to improve this paper.

\section{References}

Abt, H. A. 1984 in The MK Process and Stellar Classification, ed. R. F. Garrison,

W. W. Morgan, \& P. C. Keenan, David (Dunlap Observatory, Toronto), 340

Bohlender, D. A., Gonzalez, J.-F., \& Kennelly, E. J. 1999, A\&A, 350, 553

Breger, M. 1990, Comm. Asteroseismol., 20, 1

Breger, M. 1992, Comm. Asteroseismol., 46, 1

Breger, M., Stich, J., Garrido, R., et al. 1993, A\&A, 271, 482

Breger, M., Beck, P., Lenz, P., et al. 2006, A\&A, 455, 673

Dorokhov, N. I., \& Dorokhova, T. N. 1994, Odessa Astron. Publ., 7, 168

Dorokhova, T. N., \& Dorokhov, N. I. 1997, IBVS, 4445

Gerbaldi, M., Faraggiana, R., \& Lai, O. 2003, A\&A, 412, 447

Gray, R. O. 1988, AJ, 95, 220

Gray, R. O., \& Corbally, C. J. 1993, AJ, 106, 632

Handler, G. 1998, Baltic Astron., 7, 227

Handler, G., Shobbrook, R. R., Vuthela, F. F., et al. 2003, MNRAS, 341, 1005

Handler, G., Shobbrook, R. R., Jerzykiewicz, M., et al. 2004, MNRAS, 347, 454

Handler, G., Jerzykiewicz, M., Rodriguez, E., et al. 2006, MNRAS, 365,327

Iliev, I. K., \& Barzova, I. S. 1995, A\&A, 302, 735
Kamp, I., \& Paunzen, E. 2002, MNRAS, 335, L45

Lenz, P., \& Breger, M. 2005, Commun. Asteroseismol., 146, 53

Liu, Y. Y. 1999, IBVS, 4741

Martinez, P., Koen, C., Handler, G., \& Paunzen, E. 1998, MNRAS, 301, 1099

Mkrtichian, D. E., Kusakin, A. V., Lopez de Coca, P., et al. 2007, AJ, 134, 1713

Montgomery, M. H., \& O’Donoghue, D. 1999, Delta Scuti Star Newsletter, 13, 28 (University of Vienna)

Nielsen, R. F. 1983, Inst. Theor. Astrophys. Oslo Report No. 59, ed. O. Hauge, 141

Paunzen, E. 2004, in The A-Star Puzzle, ed. J. Zverko, J. Žižňovský, S. J. Adelman, \& W. W. Weiss, Proc. IAU Coll., 224, 443

Paunzen, E., \& Handler, G. 1996, IBVS, 4301

Paunzen, E., Iliev, I. Kh., Kamp, I., \& Barzova, I. S. 2002a, MNRAS, 336, 1030 Paunzen, E., Handler, G., Weiss, W. W., Nesvacil, N., \& Hempel, A. 2002b, A\&A, 392, 515 - PHW

Rodriguez, E., \& Breger, M. 2001, A\&A, 366, 178

Rufener, F., \& Bartholdi, P. 1982, A\&AS, 48, 503

Schneider, H. 1981, A\&AS, 44, 137

Sperl, M. 1998, Commun. Asteroseismol., 111, 1

Strassmeier, K. G., Boyd, L. J., Epand, D. H., \& Granzer, Th. 1997, PASP, 109, 697

Stürenburg, S. 1993, A\&A, 272, 139

Turcotte, S. 2002, ApJ, 573, L129

Turcotte, S. 2005, JApA, 26, 231

Turcotte, S., \& Charbonneau, P. 1993, ApJ, 413, 376

Wolf, S. C., \& Wolf, R. J. 1974, ApJ, 194, 65 